\title{
Knockdown of galectin-1 suppresses the growth and invasion of osteosarcoma cells through inhibition of the MAPK/ERK pathway
}

\author{
JIN-HAO MIAO $^{1 *}$, SHU-QIANG WANG ${ }^{1 *}$, MING-HUI ZHANG ${ }^{2}$, FENG-BIN YU $^{3}$, \\ LEI ZHANG $^{1}$, ZHONG-XIANG YU ${ }^{1}$ and YONG KUANG ${ }^{1}$
}

\author{
${ }^{1}$ Department of Orthopaedic Surgery, Shuguang Hospital, Shanghai University of Traditional Chinese Medicine, Shanghai; \\ ${ }^{2}$ Department of Medical Oncology, The Third Affiliated Hospital of Harbin Medical University, Harbin, Heilongjiang; \\ ${ }^{3}$ Department of Orthopaedic Surgery, No. 98 Hospital of PLA, Huzhou, Zhejiang, P.R. China
}

Received May 5, 2014; Accepted July 8, 2014

DOI: $10.3892 /$ or.2014.3358

\begin{abstract}
Galectin-1 (GAL1), a widely expressed $\beta$-galactoside-binding protein, exerts pleiotropic biological functions. GAL1 has been found to be upregulated in many malignancies; yet the role of GAL1 in the pathophysiology of human osteosarcoma (OS) remains uncertain. The present study was carried out to investigate the expression of GAL1 in human OS tissues and to explore its effects on the growth and invasion of OS cells. OS and corresponding adjacent non-cancerous tissues (ANCT) were collected from 30 consecutive cases. The expression of GAL1 was detected by immunohistochemical assay through tissue microarray procedure. Using small hairpin RNA (shRNA)-mediated GAL1 knockdown (Lv-shGAL1) in OS (MG-63 and U-2 OS) cells, we observed the changes in the malignant phenotype in OS cells in vitro and in vivo. As a consequence, the positive expression of GAL1 was significantly higher in OS tissues than that in the ANCT (63.3 vs. $36.7 \%, \mathrm{P}=0.029)$, and was positively correlated with distant metastasis in the OS patients $(\mathrm{P}=0.022)$. Knockdown of GAL1 suppressed cell proliferative activities and invasive potential and induced apoptosis in OS cells with decreased expression of p38MAPK, p-ERK, Ki-67 and matrix metallopeptidase-9 (MMP-9) and increased expression of caspase-3. In addition, the tumor volume in the MG-63 subcutaneous tumor models treated with Lv-shGAL1 was significantly smaller than that in the negative control (NC) group $(\mathrm{P}<0.01)$. Altogether, our findings indicate that high expression of GAL1 is associated with distant metastasis of OS patients, and knockdown of GAL1 inhibits growth and invasion of OS cells possibly through
\end{abstract}

Correspondence to: Professor Yong Kuang, Department of Orthopaedic Surgery, Shuguang Hospital, Shanghai University of Traditional Chinese Medicine, 528 Zhangheng Road, Shanghai 201203, P.R. China

E-mail: spinemiao@126.com

*Contributed equally

Key words: galectin-1, osteosarcoma, growth, invasion inhibition of the MAPK/ERK pathway, suggesting that GAL1 may represent a potential target for the treatment of cancer.

\section{Introduction}

Osteosarcoma (OS) is the most common type of primary malignant bone tumor and generally follows an aggressive clinical course (1). Although a 5-year survival rate of up to $50-70 \%$ can be achieved by using current treatment protocols, a substantial group of patients with metastatic, recurrent and/ or refractory disease remains without effective treatment options (2). A tumor is a genetic disease, which develops via a multi-step process. Multiple mutations in genes related to growth control, invasion and metastasis form the molecular genetic basis of malignant transformation and tumor progression (3). Therefore, identification of target genes involved in tumorigenesis is critical for the treatment of OS.

Galectins constitute a family of 15 mammalian galactosidebinding proteins that share a consensus amino acid sequence in their carbohydrate binding sites. They are multi-functional molecules and are expressed widely in human tumor tissues. GAL1, 3, 4, 7 and 8 are expressed in human colorectal and tongue cancer, and their expression correlates with alterations in cancer cell growth, apoptosis and cell-matrix interactions and angiogenesis, suggesting their use as biomarkers of tumor progression $(4,5)$. In addition, serum GAL2, 4 and 8 are greatly increased in colon and breast cancer patients and promote cancer cell adhesion to blood vascular endothelium (6). Proteomic analysis identified GAL1 as a predictive biomarker in classic Hodgkin lymphoma (7) and nasopharyngeal carcinoma (8).

With respect to GAL1-mediated pro-oncogene effects, it has been shown that GAL1 promotes hepatocellular carcinoma cell adhesion, polarization and in vivo tumor growth, with critical implications in liver pathophysiology (9). The expression and secretion of GAL1 can further contribute to the proliferation and invasion of pancreatic cancer cells. GAL1 may provide a novel candidate target for pancreatic cancer (10). Interestingly, the galectin-binding ability of a glycoprotein is not only a promising biomarker candidate but also may be related to the pathophysiological state of the patient (11). In addition, the interaction between cancer cells and their microenvironment is a vicious cycle that enhances 
the survival and progression of cancer, resulting in metastasis. Lung cancer-derived GAL1 was found to enhance tumorigenesis of tumor-associated dendritic cells by expressing heparin-binding EGF-like growth factor (12). GAL1 also functions as a major glycome determinant regulating Th cell development, inflammation and tumor immunity (13).

More importantly, the clinical management of OS differs significantly from that of chondrosarcoma, and it is extremely important to diagnose these two types of bone tumors accurately. Fortunately, GAL1 has been indicated as a powerful diagnostic marker that distinguishes chondroblastic OS from conventional chondrosarcomas (14). Upregulation of GAL1 may be one of the important mechanisms of bitumen-induced carcinogenic potential in human OS cells (15). However, to date, little attention has been devoted to the role and molecular mechanisms of GAL1 in the tumorigenesis of OS. Thus, in the present study, we investigated the expression of GAL1 protein in human OS using immunohistochemical (IHC) assay through a tissue microarray procedure, and explored the effects of shRNA-mediated GAL1 knockdown on the proliferative activities, invasive potential, cell apoptosis and cycle distribution in OS cells in vitro and in vivo.

\section{Materials and methods}

Materials. The human OS (MG-63 and U-2 OS) cell lines used in the experiments were from the Institute of Biochemistry and Cell Biology (Shanghai, China). Lentiviral-mediated GAL1 shRNA (Lv-shGAL1) vector, negative control vector and virion-packaging elements were from GeneChem (Shanghai, China). The primer of GAL1 was synthesized by ABI (Framingham, MA, USA). All antibodies used were purchased from Cell Signaling Technologies (Boston, MA, USA).

Drugs and reagents. Dulbecco's modified Eagle's medium (DMEM) and fetal bovine serum (FBS) were from Thermo Fisher Scientific Inc. (Waltham, MA, USA); TRIzol reagent and Lipofectamine 2000 were from Invitrogen (Carlsbad, CA, USA); M-MLV reverse transcriptase was from Promega (Madison, WI, USA); SYBR-Green Master Mixture was from Takara (Otsu, Japan). Cell apoptosis kit [propidium iodide (PI), RNase A, Annexin V-FITC] was from KeyGen Biology (Nanjing, China). The ECL-Plus kit was from GE Healthcare (Piscataway, NJ, USA).

Clinical samples and data. Human OS tissues and the corresponding adjacent non-cancerous tissues (ANCT) were obtained from 30 consecutive cases admitted to our hospital from January 2006 to December 2010. The present study was approved by the Medical Ethics Committee of Shanghai University of Traditional Chinese Medicine, and written informed consent was obtained from the patients or their parents before sample collection. Two pathologists respectively reviewed all of the cases.

Immunohistochemical staining. GAL1 antibody was used for IHC detection of protein expression in the tissue microarrays. GAL1 antibody was used at a 1:100 dilution. Endogenous peroxidase was inhibited by incubation with freshly prepared $3 \%$ hydrogen peroxide with $0.1 \%$ sodium azide. Non-specific staining was blocked with $0.5 \%$ casein and $5 \%$ normal serum. Tissue microarrays were incubated with biotinylated antibodies and horseradish peroxidase. Staining was developed with diaminobenzidine substrate and sections were counterstained with hematoxylin. Phosphate-buffered saline (PBS) replaced the GAL1 antibody in the negative controls. The expression of GAL1 was semi-quantitatively estimated as the total immunostaining scores. Expression of GAL1 in each specimen was scored according to the percentage of positively stained cells counted in five randomly selected high magnification fields: (-) no expression; (+) positive cell ratio $\leq 25 \%$; $(++)$ positive cell ratio $26-50 \%$; and $(+++)$ positive cell ratio $>50 \%$.

Cell culture and transfection. MG-63 and U-2 OS cells were cultured in DMEM supplemented with $10 \%$ heat-inactivated FBS, $100 \mathrm{U} / \mathrm{ml}$ of penicillin and $100 \mu \mathrm{g} / \mathrm{ml}$ of streptomycin. They were all placed in a humidified atmosphere containing $5 \% \mathrm{CO}_{2}$ at $37^{\circ} \mathrm{C}$. Lv-shGAL1 and the negative control virus were transfected into OS cells. Cells were subcultured at a 1:5 dilution in $300 \mu \mathrm{g} / \mathrm{ml} \mathrm{G} 418$-containing medium. Positive stable transfectants were selected and expanded for further study. The clone in which the LV-shGAL1 vector was transfected was named the Lv-shGAL1 group, and the negative control vector transfected clone was named the NC group.

Quantitative real-time PCR. To quantitatively determine the mRNA expression level of GAL1 in OS cell lines, real-time PCR was used. Total RNA of each clone was extracted with TRIzol according to the manufacturer's protocol. Reversetranscription was carried out using M-MLV, and cDNA amplification was carried out using the SYBR-Green Master Mix kit according to the manufacturer's protocol. The GAL1 gene was amplified using specific oligonucleotide primers, and the human glyceraldehyde-3-phosphate dehydrogenase (GAPDH) gene was used as an endogenous control. The PCR primer sequences were as follows: GAL1, 5'-GCGTGGCTG CTGGGAGGTATC-3' and 5'-GGAACAGAAAGACTCCA ATG-3'; $\beta$-actin, 5'-CAACGAATTTGGCT-ACAGCA-3' and 5'-AGGGGTCTACATGGCAACTG-3'. Data were analyzed using the comparative $\mathrm{Ct}$ method $\left(2^{-\Delta \Delta \mathrm{Ct}}\right)$. Three separate experiments were performed for each clone.

Western blot assay. OS cells were harvested and extracted using lysis buffer (Tris-HCl, SDS, mercaptoethanol and glycerol). Cell extracts were boiled for $5 \mathrm{~min}$ in loading buffer, and then equal amounts of cell extracts were separated on $15 \%$ SDS-PAGE gels. Separated protein bands were transferred into polyvinylidene fluoride (PVDF) membranes and the membranes were blocked in 5\% skim milk powder. The primary antibodies against p38MAPK, p-ERK, Ki-67, matrix metallopeptidase-9 (MMP-9) and caspase-3 were diluted according to the relevant instructions and incubated overnight at $4^{\circ} \mathrm{C}$. Then, horseradish peroxidase-linked secondary antibodies were added at a dilution ratio of 1:1,000, and incubated at room temperature for $2 \mathrm{~h}$. The membranes were washed with PBS for three times and the immunoreactive bands were visualized using the ECL-Plus kit according to the kit's instructions. The relative protein level in the different groups was normalized to the GAPDH concentration. Three separate experiments were performed for each clone. 
Table I. Expression of GAL1 protein in the human OS tissues.

\begin{tabular}{llcccccccr}
\hline & & & \multicolumn{3}{c}{ GAL1 protein expression (n) } & & Positive \\
\cline { 3 - 6 } Target & Group & Total & - & + & ++ & +++ & rate $(\%)$ & $\chi^{2}$ & P-value \\
\hline \multirow{2}{*}{ GAL1 } & OS & 30 & 11 & 10 & 7 & 2 & 63.3 & 4.742 & 0.029 \\
& ANCT & 30 & 19 & 7 & 4 & 0 & 36.7 & & \\
\hline
\end{tabular}

GAL1, galectin-1; OS, osteosarcoma; ANCT, adjacent non-cancerous tissues.

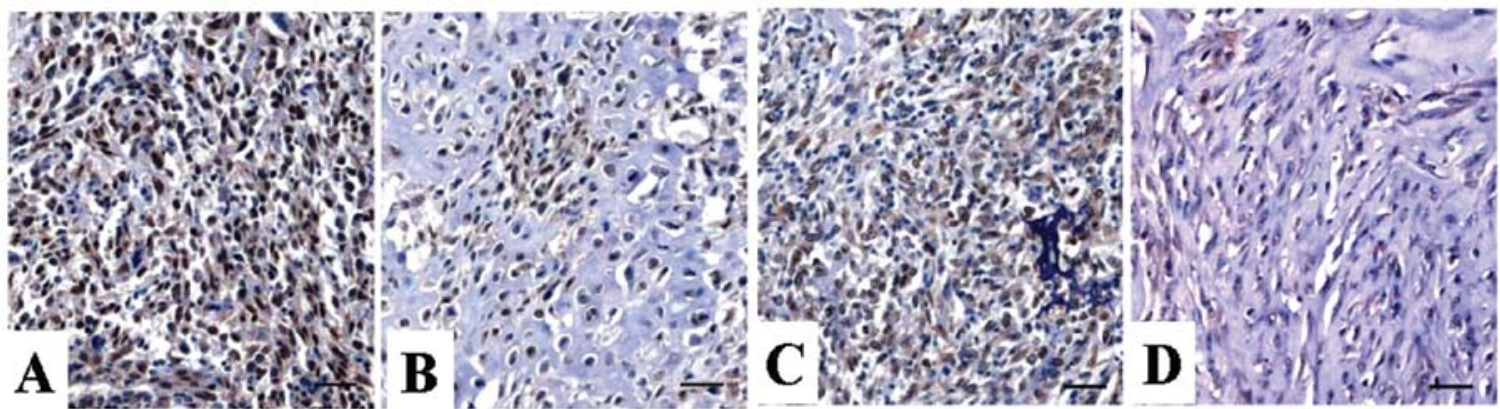

Figure 1. Expression of GAL1 protein in OS tissues (magnification, $\mathrm{x} 200$ ). OS tissues were immunohistochemically stained with an anti-GAL1 antibody. The positive expression of GAL1, mainly localized in the nucleus and cytoplasm, was increased in the OS tissues compared with the ANCT. (A) High expression in OS tissues. (B) Low expression in OS tissues. (C) High expression in ANCT tissues. (D) Low expression in ANCT tissues. GAL1, galectin-1; OS, osteosarcoma; ANCT, adjacent non-cancerous tissues.

Colony formation assay. OS cells treated with Lv-shGAL1 were counted and seeded in 12 -well plates (in triplicate) at 100 cells/well. Fresh culture medium was replaced every three days. Colonies were counted only if they contained $>50$ cells, and the number of colonies was counted from the 6th day after seeding. The cells were then stained using crystal violet. The rate of colony formation was calculated with the equation: Colony formation rate $=($ number of colonies $/$ number of seeded cells) x $100 \%$.

Transwell invasion assay. Transwell filters were coated with Matrigel (3.9 $\mu \mathrm{g} / \mu \mathrm{l}, 60-80 \mu \mathrm{l})$ on the upper surface of a polycarbonic membrane (diameter $6.5 \mathrm{~mm}$, pore size $8 \mu \mathrm{m})$. After incubation at $37^{\circ} \mathrm{C}$ for $30 \mathrm{~min}$, the Matrigel solidified and served as the extracellular matrix for analysis of tumor cell invasion. Harvested cells $\left(1 \times 10^{5}\right)$ in $100 \mu$ l of serum-free DMEM were added into the upper compartment of the chamber. Conditioned medium (200 $\mu \mathrm{l})$ derived from NIH3T3 cells was used as a source of chemoattractant, and was placed in the bottom compartment of the chamber. After a 24-h incubation at $37^{\circ} \mathrm{C}$ with $5 \% \mathrm{CO}_{2}$, the medium was removed from the upper chamber. The non-invaded cells on the upper side of the chamber were scraped off with a cotton swab. The cells that had migrated from the Matrigel into the pores of the inserted filter were fixed with $100 \%$ methanol, stained with hematoxylin, and mounted and dried at $80^{\circ} \mathrm{C}$ for $30 \mathrm{~min}$. The number of cells invading through the Matrigel was counted in three randomly selected visual fields from the central and peripheral portion of the filter using an inverted microscope (x200 magnification). Each assay was repeated three times.
Cell apoptosis analysis. To detect cell apoptosis, OS cells were trypsinized, washed with cold PBS and resuspended in binding buffer according to the instructions of the apoptosis kit. FITC-Annexin V and PI were added to the fixed cells for 20 min in darkness at room temperature. Then, Annexin $\mathrm{V}$ binding buffer was added to the mixture before the fluorescence was measured on a FACsort flow cytometer. Cell apoptosis was analyzed using Cell Quest software (Becton-Dickinson, USA). Three separate experiments were performed for each clone.

Subcutaneous tumor model and gene therapy. Six-week-old female immunodeficient nude mice (BALB/c-nu) were bred at the laboratory animal facility (Institute of Chinese Academy of Sciences, Shanghai), and were housed individually in microisolator ventilated cages with free access to water and food. Three mice were injected subcutaneously with $1 \times 10^{7} \mathrm{OS}$ cells (MG-63) in $50 \mu 1$ of PBS pre-mixed with an equal volume of Matrigel matrix (Becton-Dickinson). Mice were monitored daily and developed subcutaneous tumors. When the tumor size reached $\sim 5 \mathrm{~mm}$ in length, they were surgically removed, cut into $1-2 \mathrm{~mm}^{3}$ pieces, and re-seeded individually into other mice. When the tumor size reached $\sim 5 \mathrm{~mm}$ in length, the mice were randomly assigned to the $\mathrm{NC}$ and $\mathrm{Lv}$-shGAL1 group. In the treatment group, $15 \mu \mathrm{l}$ of Lv-shGAL1 was injected into the subcutaneous tumors using a multi-site injection format. Injections were repeated every other day after initial treatment. The tumor volume every three days was measured with a caliper, using the formula: Volume $=(\text { length } \mathrm{x} \text { width })^{2} / 2$.

Statistical analysis. SPSS 20.0 was used for statistical analysis. Kruskal-Wallis $\mathrm{H}$ and Chi-square tests were used to analyze 
A

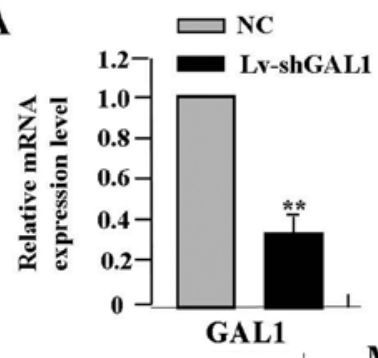

B

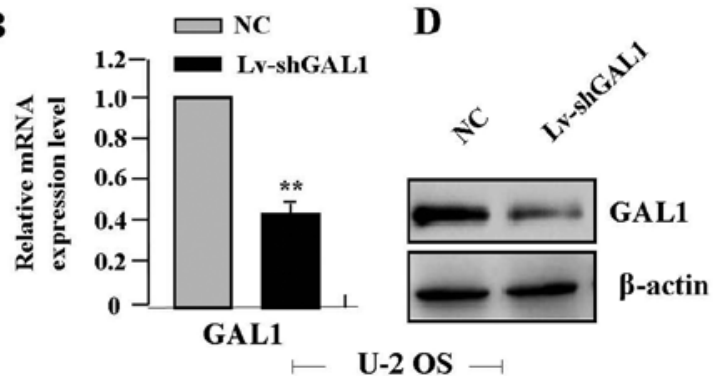

C

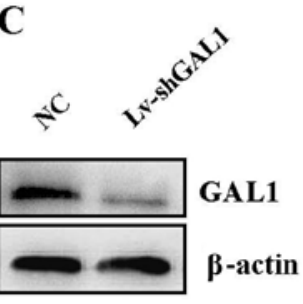

$\mathbf{E}$
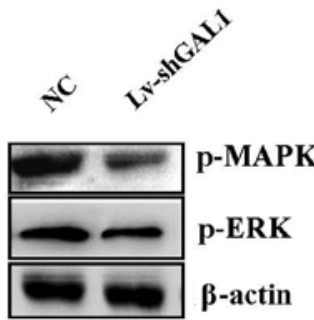

- MG-63 -

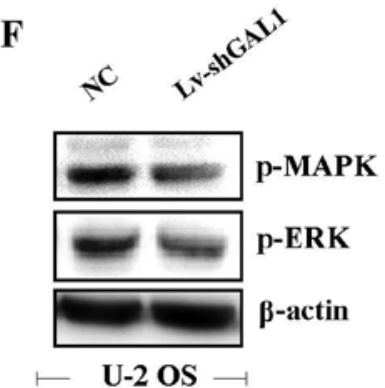

Figure 2. Knockdown of GAL1 expression in OS cells. (A and B) Real-time PCR showed a significantly lower level of GAL1 mRNA in the Lv-shGAL1 group than that in the NC group (each $\left.{ }^{* *} \mathrm{P}<0.01\right)$. (C-F) The protein expression levels of GAL1, p-MAPK and p-ERK, as indicated by western blotting, were markedly decreased in the Lv-shGAL1 group in comparison with these levels in the NC group (each $\left.{ }^{* *} \mathrm{P}<0.01\right)$. GAL1, galectin-1; OS, osteosarcoma; NC, negative control.

Table II. Correlation of GAL1 expression with clinicopathological factors of the OS patients.

\begin{tabular}{|c|c|c|c|c|}
\hline \multirow[b]{2}{*}{ Variables } & \multirow[b]{2}{*}{ Cases (n) } & \multicolumn{2}{|c|}{$\begin{array}{c}\text { GAL1 } \\
\text { expression }\end{array}$} & \multirow[b]{2}{*}{ P-value } \\
\hline & & - & + & \\
\hline Total & 30 & 11 & 19 & \\
\hline Age (years) & & & & 0.569 \\
\hline$<20$ & 21 & 7 & 14 & \\
\hline$\geq 20$ & 9 & 4 & 5 & \\
\hline Gender & & & & 0.648 \\
\hline Male & 18 & 6 & 12 & \\
\hline Female & 12 & 5 & 7 & \\
\hline Histology & & & & 0.889 \\
\hline Osteoblastic & 13 & 4 & 9 & \\
\hline Chondroblastic & 11 & 4 & 7 & \\
\hline Fibroblastic & 4 & 2 & 2 & \\
\hline Others & 2 & 1 & 1 & \\
\hline Ennecking staging & & & & 0.800 \\
\hline I & 9 & 4 & 5 & \\
\hline II & 16 & 5 & 11 & \\
\hline III & 5 & 2 & 3 & \\
\hline Distant metastasis & & & & 0.022 \\
\hline No & 11 & 7 & 4 & \\
\hline Yes & 19 & 4 & 15 & \\
\hline
\end{tabular}

GAL1, galectin-1; OS, osteosarcoma.

the expression rate in all groups. One-way analysis of variance (ANOVA) was used to analyze the differences between groups. The LSD method of multiple comparisons was used when the probability for ANOVA was statistically significant. Statistical significance was set at $\mathrm{P}<0.05$.

\section{Results}

The expression of GAL1 protein in human OS. Expression of GAL1 protein was evaluated using IHC staining. Positive expression of GAL1 protein was examined in the nucleus and cytoplasm of OS tissues and ANCT (Fig. 1), and positive GAL1 expression was detected in $63.3 \%$ (19/30) of OS tissues, compared with a positive rate of $36.7 \%$ (11/30) in ANCT $(\mathrm{P}=0.029)$ (Table I).

Correlation of GAL1 expression with clinicopathological characteristics. The association between GAL1 expression and various clinicopathological factors was analyzed. As shown in Table II, increased expression of GAL1 was closely correlated with distant metastasis of OS $(\mathrm{P}=0.022)$. However, no significant association was found between GAL1 expression and other factors including age, gender of the patients, and histology and Ennecking staging of the tumor $(\mathrm{P}>0.05$, respectively).

Knockdown of GAL1 expression in OS cells. Real-time PCR showed a significantly lower level of GAL1 mRNA in the Lv-shGAL1 group than that in the NC group (each $\mathrm{P}<0.01$ ) (Fig. 2A and B). The protein expression levels of GAL1, p-MAPK and p-ERK, as indicated by western blot assay were markedly decreased in the Lv-shGAL1 group in comparison with levels in the NC group (each $\mathrm{P}<0.01$ ) (Fig. 2C-F).

Effect of GAL1 knockdown on cell independent growth. In order to test the effect of GAL1 knockdown on cell growth, we investigated the independent growth of OS cells by colony formation assay. We found that knockdown of GAL1 significantly diminished the independent growth of OS 
A

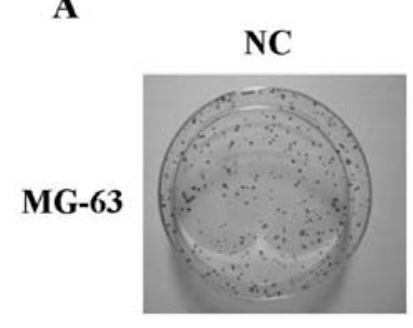

B

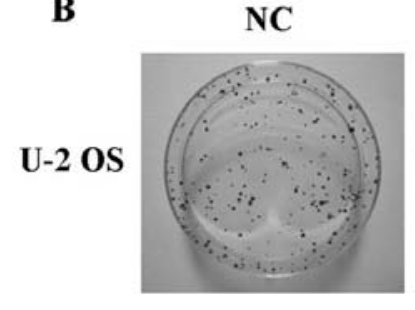

Lv-shGAL1

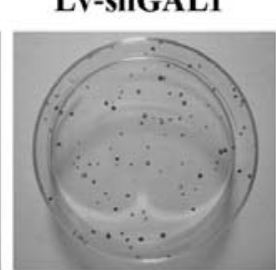

Lv-shGAL1

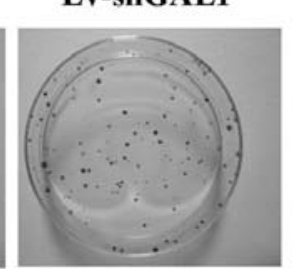

C

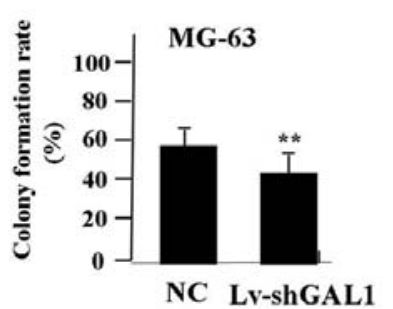

D

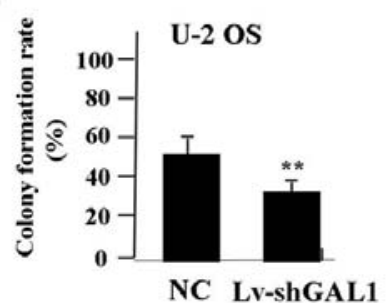

E

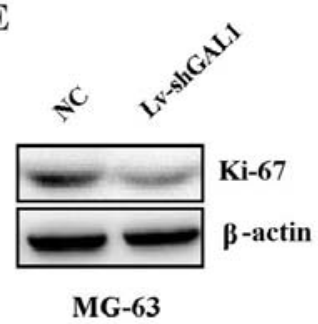

F

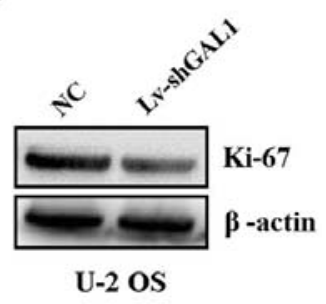

Figure 3. Effect of GAL1 knockdown on cell growth. (A-D) Colony formation assay indicated that knockdown of GAL1 significantly diminished the independent growth of OS cells when compared with the NC group. (E and F) The expression of Ki-67 protein was examined by western blotting which revealed a decreased amount of Ki-67 in the Lv-shGAL1 group compared with the NC group ("P<0.01). GAL1, galectin-1; OS, osteosarcoma; NC, negative control.

A

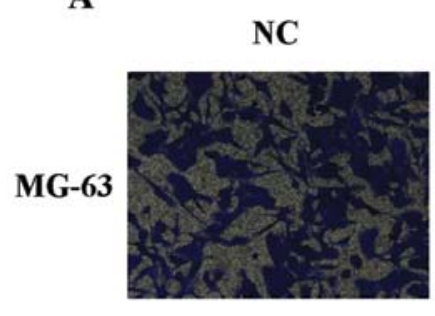

B

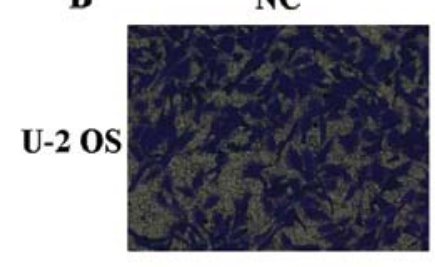

C

\section{Lv-shGAL1}

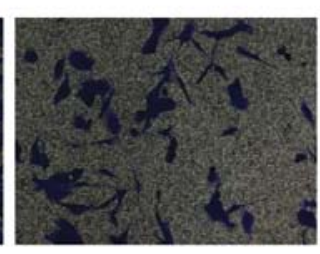

Lv-shGAL1

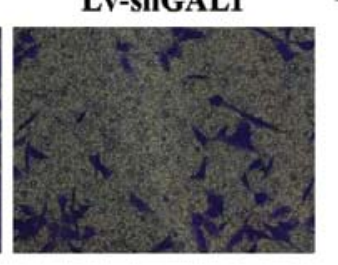

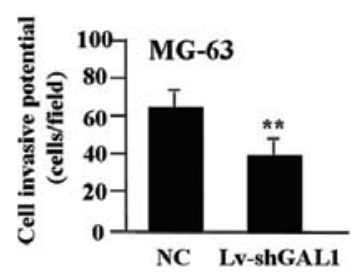

D

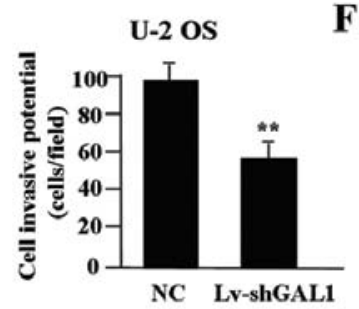

E

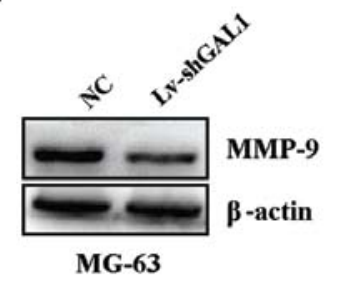

F

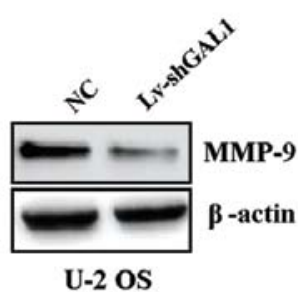

Figure 4. Effect of GAL1 knockdown on cell invasion. (A-D) The invasive potential of OS cells as indicated by Transwell assay was significantly reduced in the Lv-shGAL1 group when compared to that in the NC group $\left({ }^{* *} \mathrm{P}<0.01\right)$. (E and $\left.\mathrm{F}\right)$ The endogenous expression of MMP-9 protein, as evaluated by western blotting, was reduced in the Lv-shGAL1 group when compared with the level in the NC group (** $<<0.01)$. GAL1, galectin-1; OS, osteosarcoma; NC, negative control; MMP-9, matrix metallopeptidase-9.

cells (Fig. 3A-D, P<0.01). To determine whether knockdown of GAL1 suppressed the endogenous expression of Ki-67 through translational repression, the expression of $\mathrm{Ki}-67$ protein was examined by western blotting, indicating that Ki-67 was decreased in the Lv-shGAL1 group compared with the expression level in the NC group $(\mathrm{P}<0.01)$ (Fig. 3E and F).

Effect of GAL1 knockdown on cell invasion. To determine the effect of GAL1 knockdown on cell invasion, a Transwell assay was carried out. The invasive potential was determined on the basis of the ability of cells to invade a matrix barrier containing laminin and type IV collagen, major components of the basement membrane. Representative micrographs of Transwell filters are shown in Fig. 4A and B. The invasive activity of OS cells was significantly reduced in the Lv-shGAL1 group when compared with that in the NC group $(\mathrm{P}<0.01)$ (Fig. 4C and D). The endogenous expression of MMP-9 protein, evaluated by western blotting, was significantly reduced in the Lv-shGAL1 group when compared to that in the NC group $(\mathrm{P}<0.01)$ (Fig. 4E and F).

Effects of GAL1 knockdown on cell apoptosis. To determine whether GAL1 knockdown affects cell apoptosis, flow cytometric analysis with PI/FITC-Annexin V staining was performed. The apoptotic rate of the OS cells was markedly higher in the Lv-shGAL1 group than that in the NC group $(\mathrm{P}<0.01)$ (Fig. 5A-D). The endogenous expression of caspase-3 protein, evaluated by western blotting, was significantly increased in the Lv-shGAL1 group when compared with the expression level in the NC group $(\mathrm{P}<0.01)$ (Fig. 5E and $\mathrm{F})$. 
$\mathbf{A}$

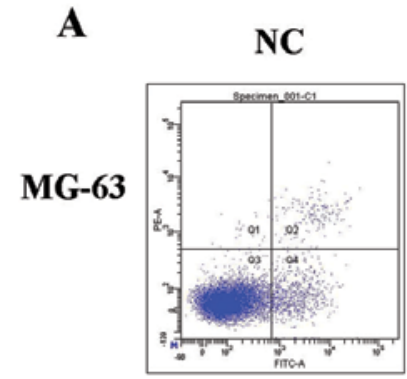

B

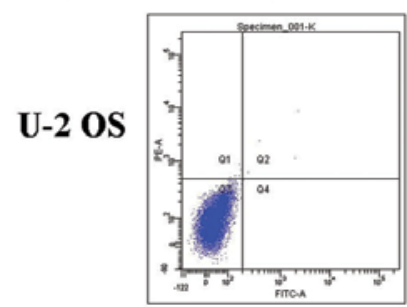

Lv-shGAL1

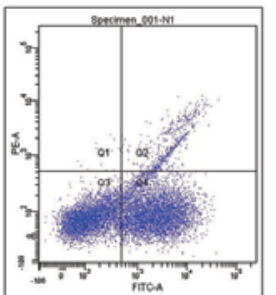

Lv-shGAL1

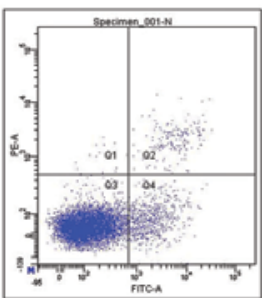

C

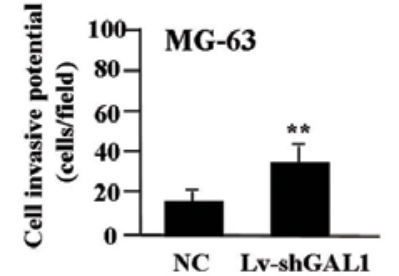

D

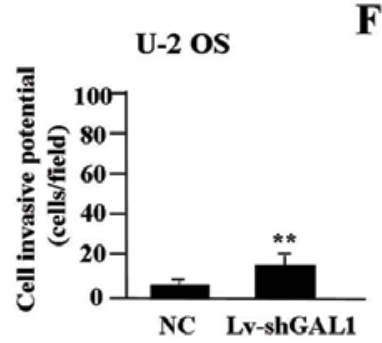

$\mathbf{E}$

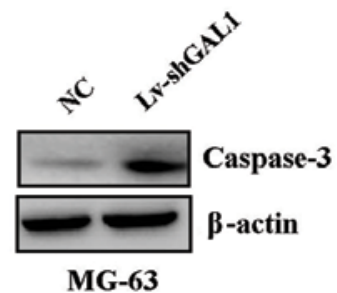

$\mathbf{F}$

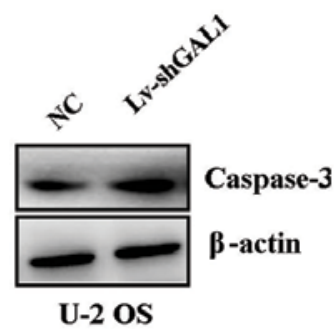

Figure 5. Effect of GAL1 knockdown on cell apoptosis. (A-D) Flow cytometric analysis showed that the apoptotic rate of the OS cells was much higher in the Lv-shGAL1 group than that in the NC group $\left({ }^{* *} \mathrm{P}<0.01\right)$. (E and F) The endogenous expression of caspase-3 protein as evaluated by western blotting was increased in the Lv-shGAL1 group when compared with the level in the $\mathrm{NC}$ group $\left({ }^{* *} \mathrm{P}<0.01\right)$. GAL1, galectin-1; OS, osteosarcoma; NC, negative control.

A

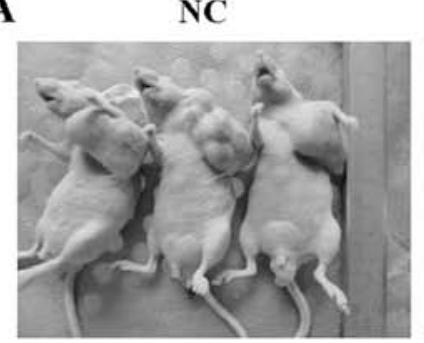

Lv-shGAL1

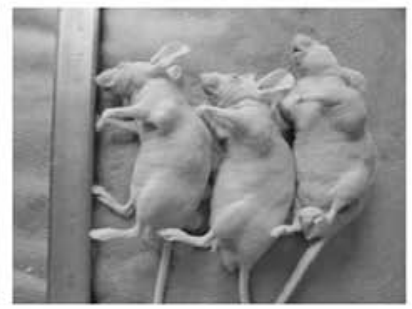

B

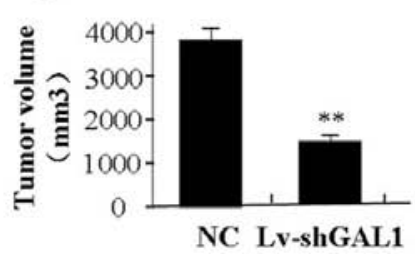

C

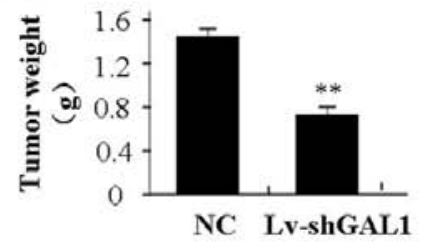

Figure 6. Effect of Lv-shGAL1 on MG-63 xenograft tumor growth. (A) The xenograft tumors treated with Lv-shGAL1 grew slower compared to those in the NC group. (B and C) The average weight and volume of the tumors were significantly smaller in the Lv-shGAL1 group than those in the NC group $\left(^{* *} \mathrm{P}<0.01\right)$. GAL1, galectin-1; NC, negative control.

Effect of GAL1 knockdown on xenograft tumor growth. Our in vitro experiments demonstrated the inhibitory effect of GAL1 knockdown on tumor growth. Thus, we further investigated the effect of GAL1 on MG-63 xenograft tumor growth in vivo. The mean volume of the tumors in the experimental mice before treatment was $75.85 \pm 19.35 \mathrm{~mm}^{3}$. During the entire tumor growth period, the tumor growth activity was assessed. The tumors treated with Lv-shGAL1 grew substantially slower when compared to the rate of growth in the NC group (Fig. 6A). When the tumors were harvested, the average weight and volume of the tumors were significantly smaller in the Lv-shGAL1 group than those of the NC group (Fig. 6B and C).

\section{Discussion}

Galectin-1 (GAL1) is a 14-kDa laminin-binding galectin involved in several biological events including regulation of tumor proliferation and metastasis. It is upregulated in human hepatocellular carcinoma (HCC), and is significantly associated with tumor invasive characteristics such as vascular invasion, suggesting it may be a new prognostic factor and a high-priority therapeutic target for HCC (16). Ovarian cancer patients with strong GAL1 peritumoral staining have poorer progression-free survival than patients with weak peritumoral staining, and inhibition of GAL1 results in the inhibition of cell growth and proliferation (17). OS is the most common malignant bone tumor in children and adolescents, unfortunately, with a poor clinical prognosis. In the present study, we found that GAL1 was highly expressed in the OS tissues compared with the ANCT, but the correlation of GAL1 expression with clinical characteristics and the poor overall survival of OS patients needs further study.

Furthermore, the primary roles of GAL1 in cancer progression and metastasis are attributed to promotion of tumor growth and angiogenesis and increased tumor cell adhesion and invasion. GAL1 plays an important role in modulating HCC cell adhesion, polarization and in vivo tumor growth with critical implications in liver pathophysiology (9). Targeting GAL1 in carcinoma-associated fibroblasts inhibits oral squamous cell carcinoma metastasis by downregulating MCP-1/CCL2 expression (18). However, some reports show 
GAL1 inhibits the viability, proliferation and Th1 cytokine production of non-malignant $\mathrm{T}$ cells in patients with leukemic cutaneous T-cell lymphoma (19), and GAL1 silencing imparts colorectal cancer with the ability to proliferate and escape apoptosis (20). Further research suggests that GAL1 plays vital pro-tumorigenic roles within the tumor microenvironment (21), and stimulates the proliferation of melanoma and neo-angiogenesis processes (22). In the present study, knockdown of GAL1 inhibited the independent growth and invasive potential, and induced apoptosis in OS cells in vitro and in vivo, suggesting that GAL1 may represent a promising and effective target for antitumor therapy.

MAPK and ERK are key regulators of oncogenic phenotypes such as proliferation, invasion, angiogenesis and inflammatory responses, which are the hallmarks of cancer. MAPK targeting inhibits proliferation, invasiveness, metastasis and drug resistance in bone sarcomas. A recent clinical trial demonstrated some clinical benefits in patients with unresectable or metastatic OS following MAPK/ERK targeting therapy (23). MAPK/ERK kinase (MEK)-phosphoinositide 3-kinase feedback signaling determines the susceptibility of breast cancer cells to MEK inhibition (24). Inhibition of PI3K/AKT and MAPK/ERK pathways leads to cell cycle arrest and apoptosis in pancreatic cancer (25). More importantly, to explore novel molecular mechanisms underlying GAL1mediated tumor progression, we analyzed the MAPK/ERK signaling pathway using PCR and western blotting. Our findings showed that knockdown of endogenous GAL1 expression in MG-63 OS cells suppressed the expression of MAPK/ERK with reduced proliferation and invasion of the tumor cells, indicating that GAL1 may affect the biological behaviors of OS cells via regulation of the MAPK/ERK signaling pathway. Chung et al (26) also suggested that p38 MAPK, ERK and COX-2 activation are novel mediators for the GAL1-promoted tumor progression and chemoresistance in lung cancer. GAL1 may be an innovative target for combined modality therapy for lung cancer.

$\mathrm{Ki}-67$ is a nuclear protein expressed in proliferating cells and is required for maintaining cell proliferation. It has been used as a marker for proliferation of OS cells (27). MMP-9 is the key enzyme involved in the degradation of type IV collagen, and a high level of MMP-9 in tissues is interrelated with tumor growth and invasion (28). In vitro knockdown of endogenous GAL1 expression in MG-63 OS cells led to a significant inhibition in the expression of Ki-67 and MMP-9, and reduced proliferative activities and invasive potential of the MG-63 cells. Hence, we speculate that GALI inhibits the proliferation and metastasis of OS cells via downregulation of the expression of Ki-67 and MMP-9.

In conclusion, our findings reveal that GAL1 is highly expressed in human OS, and is correlated with distant metastases of OS patients. Knockdown of GAL1 inhibits growth and invasion and induces apoptosis in OS cells through the MAPK/ERK pathway, suggesting that GAL1 may be a potential therapeutic target for the treatment of cancer.

\section{References}

1. Carrle D and Bielack SS: Current strategies of chemotherapy in osteosarcoma. Int Orthop 30: 445-451, 2006.
2. Bielack SS, Kempf-Bielack B, Delling G, et al: Prognostic factors in high-grade osteosarcoma of the extremities or trunk: an analysis of 1,702 patients treated on neoadjuvant cooperative osteosarcoma study group protocols. J Clin Oncol 20: 776-790, 2002.

3. Tajima Y, Yamazaki K, Makino R, et al: Gastric and intestinal phenotypic marker expression in early differentiated-type tumors of the stomach: clinicopathologic significance and genetic background. Clin Cancer Res 12: 6469-6479, 2006.

4. Barrow H, Rhodes JM and Yu LG: The role of galectins in colorectal cancer progression. Int J Cancer 129: 1-8, 2011.

5. Alves PM, Godoy GP, Gomes DQ, et al: Significance of galectins-1, $-3,-4$ and -7 in the progression of squamous cell carcinoma of the tongue. Pathol Res Pract 207: 236-240, 2011.

6. Barrow H, Guo X, Wandall HH, et al: Serum galectin-2, -4, and -8 are greatly increased in colon and breast cancer patients and promote cancer cell adhesion to blood vascular endothelium. Clin Cancer Res 17: 7035-7046, 2011.

7. Kamper P, Ludvigsen M, Bendix K, et al: Proteomic analysis identifies galectin-1 as a predictive biomarker for relapsed/ refractory disease in classical Hodgkin lymphoma. Blood 117: 6638-6649, 2011

8. Tang CE, Tan T, Li C, et al: Identification of Galectin-1 as a novel biomarker in nasopharyngeal carcinoma by proteomic analysis. Oncol Rep 24: 495-500, 2010.

9. Espelt MV, Croci DO, Bacigalupo ML, et al: Novel roles of galectin-1 in hepatocellular carcinoma cell adhesion, polarization, and in vivo tumor growth. Hepatology 53: 2097-2106, 2011.

10. Xue X, Lu Z, Tang D, et al: Galectin-1 secreted by activated stellate cells in pancreatic ductal adenocarcinoma stroma promotes proliferation and invasion of pancreatic cancer cells: an in vitro study on the microenvironment of pancreatic ductal adenocarcinoma. Pancreas 40: 832-839, 2011.

11. Carlsson $\mathrm{MC}, \mathrm{Balog} \mathrm{CI}$, Kilsgård $\mathrm{O}$, et al: Different fractions of human serum glycoproteins bind galectin-1 or galectin-8, and their ratio may provide a refined biomarker for pathophysiological conditions in cancer and inflammatory disease. Biochim Biophys Acta 1820: 1366-1372, 2012.

12. Kuo PL, Huang MS, Cheng DE, et al: Lung cancer-derived galectin-1 enhances tumorigenic potentiation of tumor-associated dendritic cells by expressing heparin-binding EGF-like growth factor. J Biol Chem 287: 9753-9764, 2012.

13. Cedeno-Laurent F, Opperman M, Barthel SR, Kuchroo VK and Dimitroff CJ: Galectin-1 triggers an immunoregulatory signature in Th cells functionally defined by IL-10 expression. J Immunol 188: 3127-3137, 2012.

14. Gomez-Brouchet A, Mourcin F, Gourraud PA, et al: Galectin-1 is a powerful marker to distinguish chondroblastic osteosarcoma and conventional chondrosarcoma. Hum Pathol 41: 1220-1230, 2010.

15. Dhondge A, Surendran S, Seralathan MV, et al: Cellular alterations and modulation of protein expression in bitumenchallenged human osteoblast cells. Environ Sci Pollut Res Int 19: 4030-4041, 2012.

16. $\mathrm{Wu} \mathrm{H}$, Chen P, Liao R, et al: Overexpression of galectin-1 is associated with poor prognosis in human hepatocellular carcinoma following resection. J Gastroenterol Hepatol 27: 1312-1319, 2012.

17. Kim HJ, Jeon HK, Cho YJ, et al: High galectin-1 expression correlates with poor prognosis and is involved in epithelial ovarian cancer proliferation and invasion. Eur J Cancer 48: 1914-1921, 2012.

18. Wu MH, Hong HC, Hong TM, et al: Targeting galectin-1 in carcinoma-associated fibroblasts inhibits oral squamous cell carcinoma metastasis by downregulating MCP-1/CCL2 expression. Clin Cancer Res 17: 1306-1316, 2011.

19. Cedeno-Laurent F, Watanabe R, Teague JE, et al: Galectin-1 inhibits the viability, proliferation, and Th1 cytokine production of nonmalignant $\mathrm{T}$ cells in patients with leukemic cutaneous T-cell lymphoma. Blood 119: 3534-3538, 2012.

20. Satelli A and Rao US: Galectin-1 is silenced by promoter hypermethylation and its re-expression induces apoptosis in human colorectal cancer cells. Cancer Lett 301: 38-46, 2011.

21. Ito K, Stannard K, Gabutero E, et al: Galectin-1 as a potent target for cancer therapy: role in the tumor microenvironment. Cancer Metastasis Rev 31: 763-778, 2012.

22. Mathieu V, de Lassalle EM, Toelen J, et al: Galectin-1 in melanoma biology and related neo-angiogenesis processes. $\mathbf{J}$ Invest Dermatol 132: 2245-2254, 2012. 
23. Chandhanayingyong C, Kim Y, Staples JR, et al: MAPK/ ERK signaling in osteosarcomas, Ewing sarcomas and chondrosarcomas: therapeutic implications and future directions. Sarcoma 2012: 404810, 2012.

24. Mirzoeva OK, Das D, Heiser LM, et al: Basal subtype and MAPK/ERK kinase (MEK)-phosphoinositide 3-kinase feedback signaling determine susceptibility of breast cancer cells to MEK inhibition. Cancer Res 69: 565-572, 2009.

25. Roy SK, Srivastava RK and Shankar S: Inhibition of PI3K/AKT and MAPK/ERK pathways causes activation of FOXO transcription factor, leading to cell cycle arrest and apoptosis in pancreatic cancer. J Mol Signal 5: 10, 2010.
26. Chung LY, Tang SJ, Sun GH, et al: Galectin-1 promotes lung cancer progression and chemoresistance by upregulating p38 MAPK, ERK, and cyclooxygenase-2. Clin Cancer Res 18: 4037-4047, 2012.

27. Tsimaratou K, Kletsas D, Kastrinakis NG, et al: Evaluation of claspin as a proliferation marker in human cancer and normal tissues. J Pathol 211: 331-339, 2007.

28. Cawston TE and Wilson AJ: Understanding the role of tissue degrading enzymes and their inhibitors in development and disease. Best Pract Res Clin Rheumatol 20: 983-1002, 2006. 